\title{
Analysis of Synaptonemal Complexes Behaviour in the Domestic Goat
}

\author{
Pavel M. Borodin', Sen-ichi Oda ${ }^{2 *}$ and Minoru Inouye ${ }^{3}$ \\ 'Institute of Cytology and Genetics, Russian Academy of Sciences, \\ Novosibirsk 630090 , Russia \\ ${ }^{2}$ Laboratory of Animal Management, School of Agricultural Sciences, Nagoya University, \\ Nagoya 464-01, Japan \\ ${ }^{3}$ Research Institute of Environmental Medicine, Nagoya University, \\ Nagoya 464-01, Japan
}

Accepted July 26, 1995

The analysis of synaptonemal complex (SC) behaviour in pachytene cells is now widely used to investigate the effects of chromosome rearrangements on fertility of laboratory and domestic animals (Chandley 1989). A more extensive work on farm animals carried out so far is on Robertsonian translocations in cattle (Switonski et al. 1987, Bouvet et al. 1990, Bouvet and Cribiu 1990a, b) and on different reciprocal translocations in pig (Gabriel-Robez et al. 1988, Jaafar et al. 1989, 1992). Yet little is known about the chromosome behaviour during meiotic prophase in the domestic goat.

The aim of our study was to analyze the pattern of chromosome pairing in male domestic goats by means of electron microscopy of surface-spread spermatocytes with special reference to the sex chromosome behaviour.

\section{Material and methods}

The authors used in this study two 4 month-old male goats of Japanese local breed (Shiba-Yagi originating from Nagasaki Prefecture, Japan) which were maintained as a SSGline at the Experimental station of Highland Animal Production, School of Agricultural Sciences, Nagoya University in Japan.

At slaughter, testes were removed and immersed into a container filled with Hanx's culture medium. Surface-spread synaptonemal complex (SC) preparations were made according to the method of our previous studies (Borodin et al. 1994a, b) described by Bouvet and Cribiu (1990a). The testis was cut open with a scalpel blade, and a small piece of tissue (about $1 \mathrm{~cm}^{3}$ ) was removed. Three drops of $0.2 \mathrm{M}$ sucrose solution were dispersed on the surface of microscopic slides precoated with $0.5 \%$ Optilux in chloroform. The tissue sample was gently pressed on the drop to spread the spermatocytes. The slides were dried out and fixed with $4 \%$ paraformaldehyde in $0.1 \mathrm{M}$ sucrose for $10 \mathrm{~min}$, washed in $0.5 \%$ Drywell solution, dried and stained with silver nitrate (Howell and Black 1980). Good spreads after light microscopic examination were transferred to specimen grids and examined and photographed with electron microscope $\mathrm{H}-500$ (Hitachi, Japan) at $75 \mathrm{kV}$.

\section{Results}

Fig. 1 shows the morphological progression of spermatocytes during prophase $I$ in the goat. In leptotene nuclei, the axial elements appear as thin, unpaired segments. In early

\footnotetext{
* Correspondence and requests for reprints.
} 


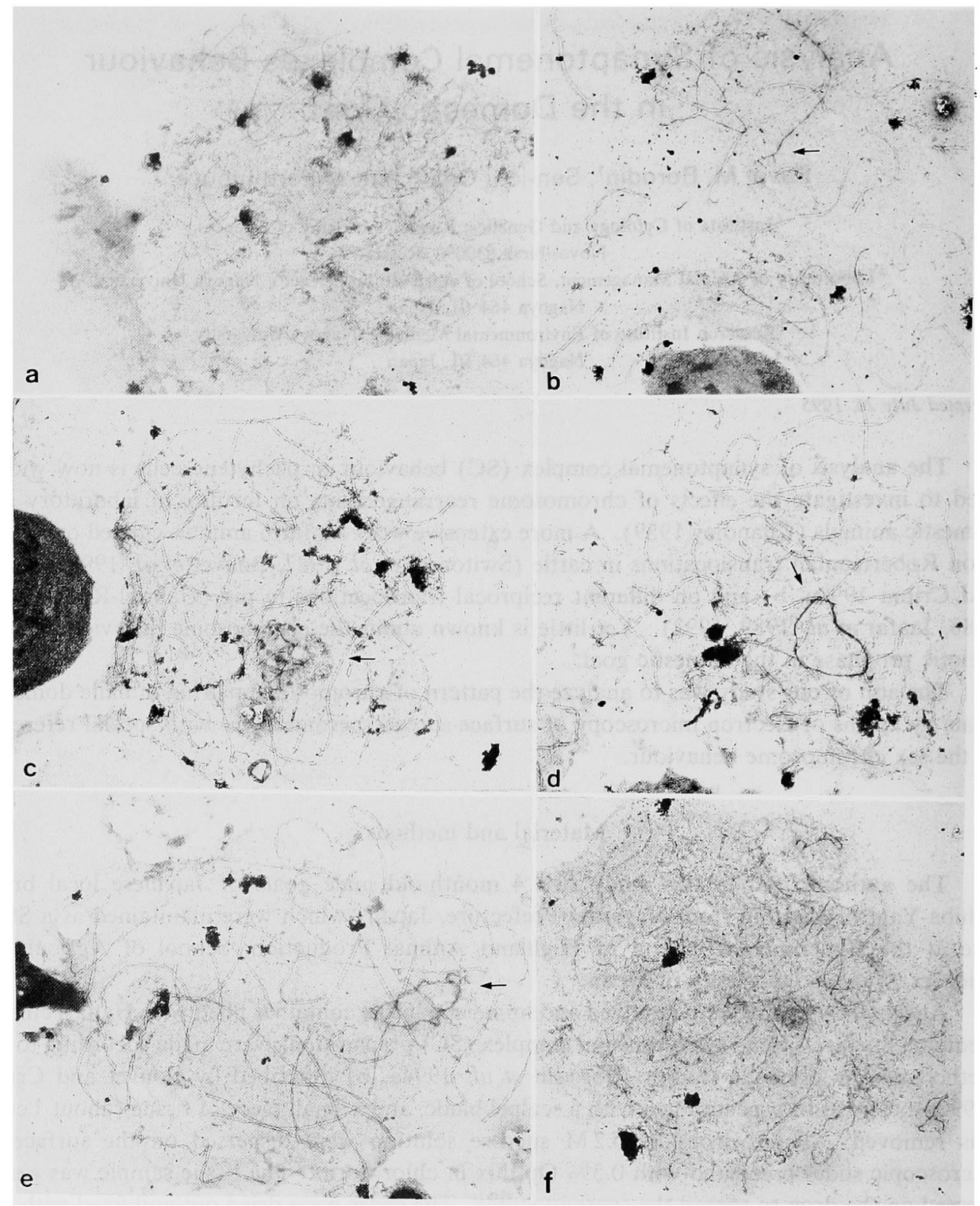

Fig. 1. Electron microphotographs of the primary spermatocytes of the domestic goat. Siver staining. a: Leptotene: the axial elements appear as thin, unpaired segments. b: Early zygotene: some of the terminal segments begin to pair. c: Mid zygotene: synapsis is still incomplete. Pairing of the axial elements produces Y-shaped or elliptical configurations. Arrow show the $\mathbf{X}$ and $Y$ axes which are recognizable as dark-staining elements. d, e: Pachytene: all 29 autosomal bivalents and the XY bivalent (arrow) are completely paired. f: Diplotene: gradual desynapsis of the lateral elements.

zygotene, some of the terminal segments begin to pair, but the assembly of the lateral elements is incomplete. The axes of the sex chromosomes cannot be identified at this stage (Fig. 1a). As zygotene progresses, the axial elements are almost completely assembled, but synapsis is still incomplete. Pairing of the axial elements starts at one or two terminal points producing 
Y-shaped or elliptical configurations. The synapsis of different autosomal bivalents is asynchronous. At the mid zygotene in the same cell, completely paired, partially paired and almost unpaired axial elements are observed. The $\mathrm{X}$ and $\mathrm{Y}$ are recognizable as dark-staining elements of different length. Interlocking of axial elements is detected at mid and late zygotene (Fig. 1b). By late zygotene, autosomal pairing is almost complete, only some ends remaining unpaired (Fig. 1c). The $\mathrm{X}$ and $\mathrm{Y}$ axes establish end-to-end association.

At pachytene, all 29 autosomal bivalents and the sex bivalent are completely paired (Fig. id, e). Nucleolar organizer regions (NOR) are observed in four to six (modal number, five) bivalents. The nucleoli display comete shape at early stages of meiotic prophase and become more round and dense during meiotic progression. All autosomal bivalents form a continuous series when arranged according to decreasing size. It is impossible to identify individual SCs.

Diplotene characterized by gradual desynapsis of the lateral elements (Fig. 1f).

The sex bivalent demonstrates a variable appearance. The SC between the $\mathrm{X}$ and $\mathrm{Y}$ once being formed remains stable in its size during pachytene. The unpaired segments of the $X$ and $\mathrm{Y}$ axes are stained darker than the paired autosomal lateral elements. In some cells they display an end-to-end association with each other. Splitting of the unpaired segments into two or more filaments is observed in one half of the analyzed pachytene cells. In some cells, the XY-pair appears as a disperse cloud, where the axial elements are hardly visible.

\section{Discussion}

Our data show general similarity between the spermatocytes of domestic goat and cattle in cell morphology and chromosome behaviour during meiotic prophase. The difference concerns the pairing pattern of the $\mathrm{X}$ and $\mathrm{Y}$ chromosomes.

According to our observations (Bitueva et al. 1994) in the cattle, the $\mathrm{X}$ and $\mathrm{Y}$ chromosomes never form true SC between each other during meiotic prophase, they establish only the end-to-end association. Usually the sex chromosomes are dissociated at pachytene. Similarly, in the cattle carrying 1; 29 Robertsonian translocation no cells with clear SC between them has been detected in the study of Switonski et al. (1987). These authors observed only a very unstable end-to-end association between $\mathrm{X}$ and $\mathrm{Y}$ axes. $\mathrm{A}$ high rate of $\mathrm{X}$ and $\mathrm{Y}$ dissociation has been also observed in cattle at metaphase diakinesis (Gustavsson 1969).

In the goat, a clear SC is formed between the $\mathrm{X}$ and $\mathrm{Y}$ axes at early pachytene when they do not display any signs of splitting. The SC remains stable during the whole pachytene. No case of $\mathrm{XY}$ separation or breaking of any axis has been detected. An explanation of this difference in $X Y$ pairing behaviour between the goat and the cattle can be drawn from a comparative analysis of the G-band pattern of their sex chromosomes (Hayes et al. 1991, Kaftanovskaya and Serov, in press).

The $\mathrm{Y}$ chromosome of the goat is metacentric and very small and of the cattle, however, it is larger and submetacentric. The goat $\mathrm{X}$ chromosome is large subtelocentric. It is suggested to be similar to the ancestral form of the Bovidae $X$ chromosome. The event providing for the transformation of the goat type into the cattle $\mathrm{X}$ was found to be a pericentric inversion of a large portion of the ancestral X including the centromere and the short arm (Evans et al. 1974, Hayes et al. 1991, Kaftanovskaya and Serov, in press). This could have lead to the transposition of the ancestral XY pairing region in the interstitial segment of the short arm of the cattle $X$ and hindered homologous pairing of sex chromosomes in male meiosis. The true pairing has been substituted by a transient end-to-end association, which is sufficient for a correct segregation. 


\section{Summary}

Synaptonemal complex analysis were carried out by electron microscopy of surface spread spermatocytes of the domestic goat. Pairing patterns of the sex chromosomes and autosomes were described.

Key words: Goat, meiosis, synaptonemal complexes, sex chromosomes.

\section{Acknowledgements}

The authors wish to thank Dr. Takagishi for her kind help in electron microscopic procedures, and Dr. O. L. Serov and Mrs. H. Kaftanovskaya for available discussing their data in press. The authors also thank K. Ohsumi, T. Tsuchiya, A. Gotoh and H. Ando for breeding and maintenance of goats at the Experimental station of Highland Animal Production, School of Agricultural Sciences, Nagoya University in Japan. A part of this research was supported by grants from the Ministry of Education, Science, Sports and Culture of Japan (S. O., No. 05680737 and No. 06680829), from the Russian National Research Program "Frontiers in Genetics" (P. B.) and the Guest Professor Program at the Research Institute of Environmental Medicine, Nagoya University in Japan (P. B.).

\section{References}

Biltueva, L., Sharshova, S., Sharshov, A., Ladygina, T., Borodin, P. and Grafodatsky, A. 1994. A new Robertsonian translocation, 8/23, in cattle. Genet. Sel. Evol. 26: 159-165.

Borodin, P., Oda, S., Takagishi, Y., Inouye, M. and Yamamura, H. 1994a. Chromosome pairing and nucleolar morphology in the male house musk shrew, Suncus murinus. Cytologia 59: 461-464.

,,,---- and $-1994 \mathrm{~b}$. Meiotic prophase in the fetal ovary of the house musk shrew, Suncus murinus. Cytologia 59: 465-469.

Bouvet, A. and Cribiu, E. P. 1990a. Synaptonemal complex behaviour in a bull homozygous for the 1; 29 Robertsonian translocation. Genet. Sel. Evol. 22: 361-366.

- and - 1990b. Analysis of synaptonemal complexes behaviour in a bull carrying the 1;29 and 9; 23 Robertsonian translocations. Reprod. Dom. Anim. 25: 215-219.

-, Popescu, C. P., De Giovanni-Macchi, A., Colombo, G. and Molteni, L. 1990. Synaptonemal complex analysis in a bull carrying a 4; 8 Robertsonian translocation. Ann. Genet. 2: 361-366.

Chandley, A. C. 1989. The handling and analysis of meiotic cells in domestic and laboratory animals, in: Halnan, C. R. E. (ed): Cytogenetics of Animals, CAB International, Wallingford, pp. 41-56.

Evans, H. J., Buckland, R. A. and Sumner, A. T. 1974. Chromosome homology and heterochromatin in goat, sheep and ox studied by banding techniques. Chromosoma 42:383-402.

Gabriel-Robez, O., Jaafar, H., Ratomponirina, C., Boscher, J., Bonneau, M., Popescu, C. P. and Rumpler, Y. 1988. Heterosynaptsis in a heterozygous fertile boar carrier a 3,7 translocation. Chromosoma 97: 26-32.

Gustavsson, I. 1969. Cytogenetics, distribution and effects of a translocation in Swedish cattle. Hereditas 63: 68-169.

Hayes, H., Petit, E. and Dutrillaux, B. 1991. Comparison of RBG-banded karytypem of cattle, sheep, and goats. Cytogenet. Cell Genet. 57: 51-55.

Howell, W. M. and Black, D. A. 1980. Controlled silver-staining of nucleolus organizer-regions with protective colloidal developer: a 1-step method. Experientia 36: 1014-1015.

Jaafar, H., Gabriel-Robez, O., Ratomponirina, C., Boscher, J., Bonneau, M., Popescu, C. P. and Rumpler, Y. 1989. Analysis of synaptonemal complexes in two fertile heterozygous boars, both carries of reciprocal translocation involving an acrocentric chromosome. Cytogenet. Cell Genet. 50: 220-225.

-, Bouvet, A., Gabriel-Robez, O., Bonneau, M., Boscher, J., Maetz, J. L., Popescu, C. P. and Rumpler, Y. 1992. Heterosynapsis in two fertile but hypoprolific boars carriers of reciprocal translocations. Ann. Genet. 35: 37.

Kaftanoyskaya, H. M. and Serov, O. L. High resolution GTG-banded chromosomes of cattle, sheep, and goat: A comparative study. J. Hered. (in press).

Switonski, M., Gustavsson, I. and Ploen, L. 1987. The nature of the 1; 29 translocation in cattle as revealed by synaptonemal complex analysis using electron microscopy. Cytogenet. Cell Genet. 44: 103-111. 